\title{
Caracterização Antropométrica de Portadores de Doença Renal em Tratamento Conservador
}

Anthropometric Characterization of Renal Disease Patients in Conservative Treatment

Caracterización Antropométrica de Portadores de Enfermedad Renal en Tratamiento Conservador

Erminia Luzia da Silva Marinho ${ }^{1}$

Rayne Gomes Amorim ${ }^{2}$

Juliana Célia de Farias Santos ${ }^{3}$

\section{Resumo}

Objetivo: Traçar o perfil antropométrico de pacientes portadores de DRC em tratamento conservador e avaliar a associação com o estadiamento da DRC em um centro de nefrologia num hospital universitário. Método: Estudo transversal com pacientes de ambos os sexos e idade superior a 18 anos atendidos pelo ambulatório de nutrição.

Os dados antropométricos coletados foram peso, altura e circunferência da cintura (CC) e os respectivos índices e percentuais calculados: índice de massa corporal (IMC) e \% de gordura corporal (\%GC). A taxa de filtração glomerular (TFG) foi estimada pela fórmula CKDEPI. Os dados foram analisados com o programa Stata versão 13.0. Resultados: Compuseram a amostra 42 pacientes, $26,1 \%$ nos estágios 1 e 2 , e 59,3\% nos estágios 3,4 e 5 da DRC. As médias gerais de IMC, CC e \%GC foram $27,4 \pm 6,2 \mathrm{~kg} / \mathrm{m}^{2}, \quad 96,55 \pm 14,13 \mathrm{~cm}$, $35,7 \pm 9,5 \%$, respectivamente. Foi

${ }^{1}$ Nutricionista. Graduada pela Universidade Federal de Alagoas. Residente Uniprofissional pelo Instituto de Medicina Integral Professor Fernando Figueira - IMIP/PE. Autora correspondente: Rua Jorge de Lima, $\mathrm{n}^{\mathrm{o}}$ 245. APT 801 TORRE Alegro. Imbiribeira. 51160-070. Recife, PE, Brasil. E-mail: erminiamarinho2008@hotmail.com

${ }^{2}$ Nutricioinista. Graduada pela Universidade Federal de Alagoas. Mestranda em Nutrição pelo Programa de Pós-Graduação em Nutrição da Universidade Federal de Alagoas.

${ }^{3}$ Nutricionista. Graduada pela Universidade Federal de Alagoas. Professora da Faculdade de Nutrição (FANUT) da Universidade Federal de Alagoas.

Recebido: Abr./2018 - Aceito: Ago./2018.

Revist. Port.: Saúde e Sociedade. 2018;3(2):779-793. 
encontrada diferença estatisticamente significativa $(\mathrm{p}<0,05)$ para variáveis antropométricas, bioquímicas e TFG entre os grupos com \%GC adequado e alto. Conclusão: A maior parcela da amostra estava em estágios finais da DRC e o excesso de peso foi mais prevalente. Contudo, não foi possível avaliar o perfil antropométrico por estadiamento da DRC, tal fato justificado pelo tamanho da amostra, nosso fato limitante.

\section{Descritores: Estado Nutricional;} Antropometria; Insuficiência Renal Crônica.

\section{Abstract}

Objective: To outline the anthropometric profile of patients with $C K D$ in conservative treatment and to evaluate the association with CKD staging in a nephrology center in a university hospital. Method: Crosssectional study with patients of both sexes and over 18 years of age attended by the nutrition clinic. The anthropometric data collected were weight, height and waist circumference (WC) and the respective indexes and percentages calculated: body mass index (BMI) and\% body fat (\% GC). The glomerular filtration rate (GFR) was estimated by the formula CKD-EPI. The data were analyzed with the program Stata version 13.0. RESULTS: The sample comprised 42 patients, $26.1 \%$ in stages 1 and 2, and 59.3\% in stages 3,4 and 5 of CKD. The mean means of BMI, CC and\% GC were 27.4 $\pm 6.2 \mathrm{~kg} / \mathrm{m}^{2}, 96.55 \pm 14.13 \mathrm{~cm}, 35.7 \pm$ $9.5 \%$, respectively. A statistically significant difference $(p<0.05)$ was found for anthropometric, biochemical and GFR variables between the groups with adequate and high GC\%. Conclusion: The largest portion of the sample was in the final stages of CKD and overweight was more prevalent. However, it was not possible to evaluate the anthropometric profile by staging of CKD, such fact justified by the size of the sample, our limiting fact.

\section{Descriptors: Nutritional Status; Anthropometry; Chronic Renal Insufficiency.}

\section{Resumen}

Objetivo: $\quad$ Trazar el perfil antropométrico de pacientes portadores de DRC en tratamiento conservador $y$ evaluar la asociación con la estadificación de la DRC en un centro de nefrología en un hospital universitario. Método: Estudio 
transversal con pacientes de ambos sexos y edad superior a 18 años atendidos por el ambulatorio de nutrición. Los datos antropométricos recogidos fueron peso, altura $y$ circunferencia de la cintura (CC) y los respectivos indices y porcentajes calculados: índice de masa corporal (IMC) $y \%$ de grasa corporal (\% GC). La tasa de filtración glomerular (TFG) fue estimada por la fórmula CKD-EPI. Los datos se analizaron con el programa Stata versión 13.0. Resultados: Compusieron la muestra 42 pacientes, 26,1\% en las etapas 1 y 2, y $59,3 \%$ en las etapas 3,4 y 5 de la DRC. Las medias generales de IMC, CC $y \%$ GC fueron $27,4 \pm 6,2 \mathrm{~kg} / \mathrm{m}^{2}, 96,55 \pm$ $14,13 \mathrm{~cm}, 35,7 \pm 9,5 \%$, respectivamente. Se encontró diferencia estadísticamente significativa $(p<0,05)$ para variables antropométricas, bioquímicas y TFG entre los grupos con\% GC adecuado y alto. Conclusión: La mayor parte de la muestra estaba en etapas finales de la DRC y el exceso de peso fue más prevalente. Sin embargo, no fue posible evaluar el perfil antropométrico por estadificación de la DRC, tal hecho justificado por el tamaño de la muestra, nuestro hecho limitante.

\section{Descriptores: Estado Nutricional, Antropometría, Insuficiencia Renal Crónica}

\section{Introdução}

As doenças crônicas não transmissíveis (DCNT) atualmente são a maior causa de morbimortalidade no mundo e no Brasil. A mudança no perfil de mortalidade dos últimos anos atribui as DCNT $67 \%$ dos óbitos ocorridos no mundo em 2012 e $74 \%$ dos óbitos ocorridos no Brasil em 2015(1,2). E, segundo a $\mathrm{WHO}^{(1)}$, o número total de mortes no mundo por DCNT aumentará 14 milhões nos próximos 13 anos.

Associado ao crescimento das DCNT, em especial a hipertensão arterial sistêmica (HAS) e diabetes mellitus (DM), o excesso de peso vem se tornando uma pandemia mundial ${ }^{(3)}$. Dados da última pesquisa em vigilância de fatores de risco e proteção para doenças crônicas por inquérito telefônico $\quad$ (VIGITEL) $^{(4)}, \quad 72,7 \%$ da população brasileira tem excesso de peso. Atualmente a HAS, DM e obesidade são as principais causas para a doença renal crônica $(\mathrm{DRC})^{(5-7)}$.

A DRC é mais um problema crescente de saúde pública ${ }^{(3)}$. É caracterizada pela perda lenta e progressiva das funções endócrinas, 
glomerulares e tubulares por um período superior a três meses, onde o controle metabólico e hidroeletrolítico não são mantidos em homeostase ${ }^{(8)}$.

Condições extremas do estado nutricional se traduzem em prognósticos negativos na fase não dialítica. Ou seja, tanto a desnutrição energético-proteica como o excesso de peso podem trazer danos irreversíveis ao paciente com $\mathrm{DRC}^{(5)}$. A manutenção de um adequado estado nutricional é imprescindível, onde a intervenção nutricional pode promover uma redução do declínio da taxa de filtração glomerular (TFG), melhor controle das comorbidades e, evitar complicações como hiperfosfatemia, hipercalemia e acidose metabólica ${ }^{(9)}$.

Contudo, para um adequado planejamento dietético, um correto diagnóstico nutricional é necessário, e para isso, a avaliação nutricional é imprescindível. A antropometria vem como um método de alta reprodutibilidade e baixo custo e como um bom preditor das condições nutricionais e de saúde ${ }^{(10)}$.

Portanto, o objetivo desse trabalho foi traçar o perfil antropométrico de pacientes portadores de DRC em tratamento conservador em um centro de nefrologia num Hospital Universitário.
Método

Estudo epidemiológico com delineamento transversal e descritivo que fez parte de uma pesquisa maior intitulada: Caracterização, intervenção nutricional e desfechos clínicos de portadores de doença renal crônica em fase não-dialítica acompanhados pelo ambulatório de nutrição em nefrologia do Hospital Universitário Professor Alberto Antunes, Maceió- Alagoas, realizado no Centro Integrado de Nefrologia de um Hospital Universitário, com objetivo foi traçar o perfil antropométrico de pacientes portadores de DRC em tratamento conservador e avaliar a associação desse com o estadiamento da DRC.

O cálculo amostral foi realizado com auxílio do programa Statcalc do Epi Info versão 7.0, considerando como desfecho de interesse a doença renal crônica, segundo Barros et al. ${ }^{(11)}$ estimado em 1,25\%, considerando um erro amostral de 3\%, um nível de confiança de $99 \%$ e adicionado $10 \%$ para eventuais perdas seriam necessários 100 voluntários.

A amostra foi composta por indivíduos maiores de 18 anos de ambos os sexos e portadores de DRC em fase não dialítica nos estágios 1-5 com TFG $\geq 90$ e $>15 \mathrm{~mL} / \mathrm{min} / 1,73 \mathrm{~m}^{2}$, sendo 
excluídos as gestantes, alcoólatras, portadores de HIV e câncer.

A coleta de dados ocorreu no período de agosto de 2016 a abril de 2017. As consultas eram guiadas por meio de um formulário padronizado, os dados coletados e os cálculos dos respectivos índices, adequações e porcentagens obtidos posteriormente estão abaixo descritos:

Dados Socioeconômicos: Foram coletados dados referentes à escolaridade, estado civil, idade, ocupação e renda familiar.

Dados clínicos: Foi coletada a partir dos prontuários da nefrologia toda história clínica do paciente, doenças de base pré-existentes a DRC e sintomatologia clínica. Quando os dados não eram obtidos nos prontuários, os mesmos eram coletados durante as consultas.

Dados antropométricos: Medidas de peso, altura e da circunferência da cintura foram realizadas com os pacientes em pé, descalços, com os calcanhares juntos, costas retas e com a cabeça no plano de Frankurt, com exceção da estatura em idosos, que foi realizada de modo diferente e descrita a seguir. O Peso $(\mathrm{Kg})$ - foi obtido através de balança digital Fillizola, com capacidade de $150 \mathrm{~kg}$ e com precisão de 100g; Estatura (m) - Nos adultos foi obtida através de estadiômetro fixo acoplado à balança digital com precisão de $0,5 \mathrm{~cm}$ e nos idosos a estatura foi estimada por meio da altura do joelho (AJ). Para isso, com o idoso sentado e com o joelho flexionado em ângulo de $90^{\circ}$, era aferido o comprimento entre o calcanhar e a superfície anterior da perna na $\mathrm{AJ}$, medido com fita métrica inextensível com precisão de $1 \mathrm{~cm}$. A altura foi estimada utilizando as equações preditivas de Chumlea de 1988 ${ }^{(12)}$; O índice de Massa Corporal (IMC) $\left(\mathrm{kg} / \mathrm{m}^{2}\right)$ - foi obtido pela razão peso, $(\mathrm{kg})$ altura $(\mathrm{m})$ ao quadrado e foram classificados segundo as recomendações da $\mathrm{OMS}^{(13)} 1995$ para adultos e Lipschitz ${ }^{(14)}$ para idosos. Circunferência do Braço (CB) (cm) Foi obtida através da medida realizada com fita métrica inextensível medindo o braço flexionado em direção ao tórax, formando um ângulo de $90^{\circ}$, marcando o ponto médio, encontrado entre $\mathrm{o}$ acrômio e o olecrano. Após a marcação, os pacientes foram orientados a ficarem em posição fundamental. O resultado obtido foi avaliado de acordo com Frisancho $1974^{(15)}$, utilizando-se como referência 0 percentil $50^{\circ}$ para população brasileira, com a equação: adequação da $\mathrm{CB}(\%)=\mathrm{CB}$ obtida $(\mathrm{cm})$ x 100 / CB percentil 50. Circunferência Cintura $(\mathrm{CC})(\mathrm{cm})-$ Foi realizada com 
fita métrica inextensível (precisão de $0,1 \mathrm{~cm}$ ), sendo medido o ponto médio entre a última costela e a crista ilíaca. Foi classificada de acordo com Brandão et $\mathrm{al}^{(16)}$; A porcentagem de gordura corporal $(\% \mathrm{GC})$ foi calculada pela fórmula proposta por Deurenberg et al. ${ }^{(17)}: \% \mathrm{GC}=(1,2 \mathrm{x}$ IMC $)+(0,23 \mathrm{x}$ idade) - (10,8 x sexo) - 5,4, onde o IMC é expresso em $\mathrm{kg} / \mathrm{m}^{2}$, idade em anos e sexo feminino $=0$ e sexo masculino $=1$. As $\% \mathrm{GC}$ foram classificadas como alta quando acima de $25 \%$ nos homens e de $32 \%$ nas mulheres, de acordo com Lohman $^{(18)}$.

Dados bioquímicos: Estes foram solicitados quando na ausência destes em prontuário nos últimos três meses: ureia e creatinina. Os parâmetros de referência para a bioquímica foram considerados aqueles utilizados pelo laboratório de análises clínicas do hospital.

O método utilizado para calcular a TFG foi a fórmula proposta por CKDEPI composto por creatinina sérica, idade e sexo, preconizado pelo Ministério da Saúde ${ }^{(19)}$ e os estágios da DRC classificado pelo KDIGO $2017^{(8)}$.

O banco de dados foi construído no software Microsoft Office Excel. Na análise descritiva, as variáveis contínuas e categóricas foram expressas por meio de média, desvio-padrão e porcentagens, respectivamente. As variáveis categóricas foram comparadas utilizando o teste qui-quadrado. Foram aplicados os testes de shapiro wilk e Levene para avaliar a normalidade e homocedasticidade das distribuições, respectivamente. Para a comparação entre os grupos com adequado e alto \%GC entre as variáveis antropométricas e bioquímicas foi aplicado o teste de U de Mann-Whitney. Todas as análises foram realizadas com o auxílio do software Stata (Statistics/Data Analysis) versão 13.0, adotando um nível de confiança de 95\% $(\alpha=0,05) . \quad \mathrm{O} \quad$ valor de $\quad \mathrm{p}<0,05$ foi estatisticamente significativo.

O presente trabalho foi aprovado pelo Comitê de Ética em Pesquisa da Universidade Federal de Alagoas, e todos os participantes foram informados e incluídos neste estudo após assinarem o TCLE. O projeto do presente estudo foi aprovado sob o parecer (CAAE 43975115.9.0000.5013).

\section{Resultados}

A amostra foi composta por 42 pacientes, sendo $57,14 \%$ adultos com média de idade entre $56 \pm 15$ anos, 50\% do sexo feminino. Os casados compunham $50 \%$ da amostra e $47,62 \%$ declararam possuir vínculo 
empregatício. A renda familiar média era de dois salários mínimos, com média per capita de $\mathrm{R} \$ 630$ reais e apenas 1 paciente estava abaixo da linha de pobreza. Do total de pacientes, $59,52 \%$ residiam na capital e $66,67 \%$ eram naturais do interior.

Em relação ao nível de escolaridade, a maior prevalência foram daqueles que tinham o Ensino Fundamental incompleto, perfazendo
45,24\% da amostra. Apenas 1 paciente, o que representa $2,38 \%$, afirmou ter Ensino Superior completo, sobre o sistema que dispunham para acesso à saúde, $85,71 \%$ relataram que a única forma de assistência era o Sistema Único de Saúde (SUS). Os demais dados sobre o perfil socioeconômico e demográfico dos pacientes estão dispostos na tabela 1.

\section{Tabela 1: Perfil Socioeconômico Demográfico dos Pacientes em Tratamento Conservador}

\begin{tabular}{cccc}
\hline Variável & Classificação/Condição & N (42) & \% (100) \\
\hline Sexo & Masculino & $21 / 42$ & 50 \\
& Feminino & $21 / 42$ & 50 \\
Faixa etária & Adulto & $24 / 42$ & 57,1 \\
& Idoso & $18 / 42$ & 42,9 \\
Estado Civil & Solteiro & $15 / 41$ & 35,7 \\
& Casado & $21 / 41$ & 50 \\
Naturalidade & Viúvo & $5 / 41$ & 11,9 \\
& Cidade & $10 / 38$ & 23,8 \\
Procedência & Interior & $28 / 38$ & 66,7 \\
& Cidade & $25 / 41$ & 59,5 \\
Escolaridade & Interior & $16 / 41$ & 38,1 \\
& Analfabeto & $3 / 41$ & 7,1 \\
& Ensino Fundamental Incompleto & $19 / 41$ & 45,2 \\
& Ensino Fundamental Completo & $4 / 41$ & 9,5 \\
& Ensino Médio Incompleto & $5 / 41$ & 11,9 \\
Acesso aos serviços de saúde & Ensino Médio Completo & $8 / 41$ & 19,1 \\
& Superior Incompleto & $1 / 41$ & 2,4 \\
Vínculo empregatício & Superior Completo & $1 / 41$ & 2,4 \\
& Sú & $36 / 40$ & 85,7
\end{tabular}

Quanto a classificação pelos estágios da DRC, 26,19\% estavam nos estágios 1 e $2 ; 42,85 \%$ nos estágios $3 \mathrm{e}$ 4 e $16,67 \%$ no estágio final da fase não dialítica. No que se refere às comorbidades, $88,1 \%$ tinham HAS e 61,9\% tinham DM. Sendo encontrados em nossa amostra $54,76 \%$ de indivíduos que apresentavam as duas comorbidades associadas, e daqueles que 
apresentavam alguma doença cardiovascular (DCV), apenas 23,81\% confirmaram a existência desta antes do diagnóstico de DRC.
Dados adicionais sobre a caracterização clínicas, fatores de riscos e associados à DRC dos pacientes estão na tabela 2 .

Tabela 2: Caracterização Clínica Dos Pacientes Em Tratamento Conservador

\begin{tabular}{cccc}
\hline Variável & Características & N (42) & \% (100) \\
\hline Tabagismo & Tabagista & $3 / 40$ & 7,1 \\
& Ex Tabagista & $13 / 40$ & 30,9 \\
Etilismo & Etilista & $10 / 39$ & 23,8 \\
& Ex Etilista & $12 / 38$ & 28,6 \\
Função gastrointestinal & Regular & $29 / 39$ & 69,1 \\
& Irregular & $10 / 39$ & 23,8 \\
HAS & Sim & $37 / 42$ & 88,1 \\
& Não & $5 / 42$ & 11,9 \\
DM & Sim & $26 / 42$ & 61,9 \\
& Não & $16 / 42$ & 38,1 \\
HAS+DM & Sim & $23 / 42$ & 54,8 \\
& Não & $19 / 42$ & 45,2 \\
DCV Pré Existente & Sim & $10 / 36$ & 23,8 \\
& Não & $26 / 36$ & 61,9 \\
Condição da urina & Presença de espuma & $24 / 39$ & 57,1 \\
& Alteração na coloração & $30 / 39$ & 71,4 \\
Uremia & Sim & $15 / 29$ & 35,7 \\
& Não & $14 / 29$ & 33,3 \\
Estágio da DRC & Estágio 1 & $6 / 36$ & 14,3 \\
& Estagio 2 & $5 / 36$ & 11,9 \\
Mellitus; DCV = Doença Cardiovascular; DRC & D Doença Renal Crônica. & 4,8 \\
& Estágio 3 A & $2 / 36$ & 9,5 \\
& Estágio 3 B & $4 / 36$ & 28,6 \\
& Estágio 4 & $12 / 36$ & 16,7 \\
& Estágio 5 & $7 / 36$ & \\
& & &
\end{tabular}

Quanto a classificação pelos estágios da DRC, 26,19\% estavam nos estágios 1 e 2; $42,85 \%$ nos estágios 3 e 4 e $16,67 \%$ no estágio final da fase não dialítica. No que se refere às comorbidades, $88,1 \%$ tinham HAS e $61,9 \%$ tinham DM. Sendo encontrados em nossa amostra $54,76 \%$ de indivíduos que apresentavam as duas comorbidades associadas, e daqueles que apresentavam alguma doença cardiovascular (DCV), apenas 23,81\% confirmaram a existência desta antes do diagnóstico de DRC.

Dados adicionais sobre a caracterização clínicas, fatores de riscos e associados à DRC dos pacientes estão na tabela 2 . 
Tabela 3: Médias Gerais das Medidas Antropométricas, IMC e Percentuais de CB e GC dos Pacientes em Tratamento Conservador

\begin{tabular}{|c|c|c|c|c|c|c|c|}
\hline Va & $\begin{array}{r}\text { Peso } \\
\text { (kg) }\end{array}$ & $\begin{array}{c}\text { Estatura } \\
(\mathrm{m})\end{array}$ & $\begin{array}{c}\text { IMC } \\
\left(\mathrm{kg} / \mathrm{m}^{2}\right)\end{array}$ & $\begin{array}{c}\mathrm{CB} \\
(\mathrm{cm})\end{array}$ & $\begin{array}{c}\% \text { CB } \\
(\%) \\
\end{array}$ & $\begin{array}{c}\mathrm{CC} \\
(\mathrm{cm})\end{array}$ & $\begin{array}{c}\% \text { GC } \\
(\%)\end{array}$ \\
\hline Médi & $\begin{array}{l}70.5 \pm \\
15,23\end{array}$ & $\begin{array}{c}1.60 \pm \\
0,08\end{array}$ & $\begin{array}{c}27.42 \pm \\
6,25\end{array}$ & $\begin{array}{c}30,85 \pm \\
4,99\end{array}$ & $\begin{array}{c}100.15 \pm \\
16,02\end{array}$ & $\begin{array}{c}96,55 \pm \\
14,13\end{array}$ & $\begin{array}{c}35.65 \pm \\
9,48\end{array}$ \\
\hline \multicolumn{8}{|c|}{$\begin{array}{l}\mathrm{DP}=\text { Desvio Padrão; } \%=\text { Percentual } \text { IMC = Índice de Massa Corporal; } \mathrm{CB}=\text { Circunferência Braço; \% } \\
\mathrm{CB}=\text { Percentual de Adequação da Circunferência Braço; } \mathrm{CC}=\text { Circunferência da Cintura; \% GC = } \\
\text { Percentual de Gordura Corporal. }\end{array}$} \\
\hline
\end{tabular}

A avaliação bioquímica revelou que os marcadores de função renal, creatinina e ureia, apresentaram média de $2,58 \pm 1,7 \mathrm{mg} / \mathrm{dL} \quad$ e $94,4 \pm$ $54,69 \mathrm{mg} / \mathrm{dL}$, respectivamente, e a TFG média foi de 44,18 $\pm 39,04$ $\mathrm{mL} / \mathrm{min} / 1,73 \mathrm{~m}^{2}$.

Não foram observadas associações entre os estágios de DRC e as variáveis antropométricas, contudo, quando comparadas às médias das variáveis antropométricas $(\mathrm{IMC}, \mathrm{CB}$, \%CB), creatinina e $\mathrm{TFG}$ em relação aos grupos com adequado e alto $\% \mathrm{GC}$, foi verificado diferença estatisticamente significativa $(\mathrm{p}<$ $0,05)$, como exposto na tabela 4 .

Tabela 4: Comparação das Médias das Variáveis Antropométricas, Bioquímica e da TFG em Relação aos Grupos com Adequado e Alto \%GC

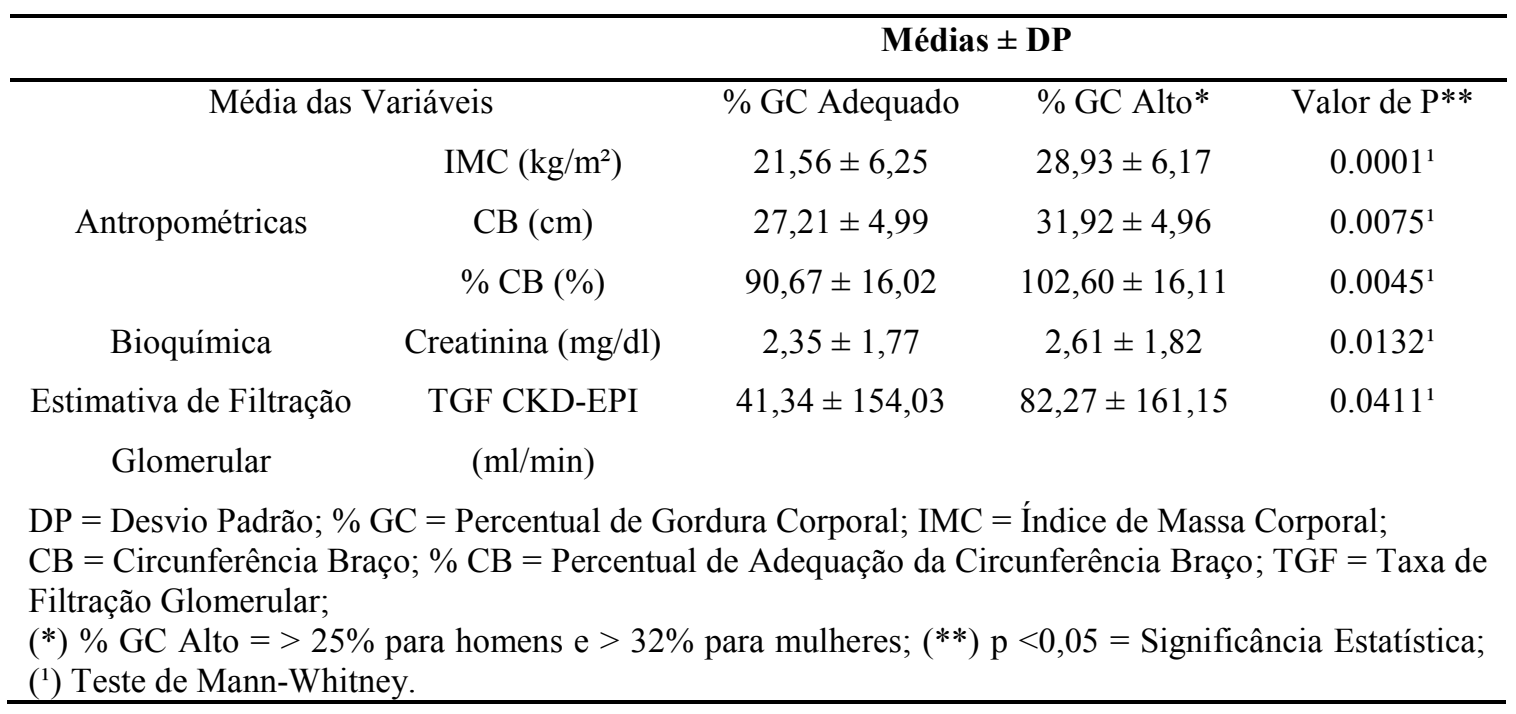

\section{Discussão}

Com a mudança no perfil da morbimortalidade no Brasil e no mundo, onde são atribuídas as DCNT as maiores frequências de morte prematura, é possível que essa mudança tenha proporcionado o perfil de 
pacientes com DRC em nosso estudo, onde a maior prevalência foi em adultos, esse novo perfil é marcado pelo aumento exponencial da obesidade, HAS e DM, que são as principais comorbidades associadas ao desenvolvimento da DRC, principalmente quando não controladas $^{(5-7)}$.

Numa coorte realizada com afroamericanos com DRC hipertensiva, desemprego, renda limitada e a baixa escolaridade foram fatores associados diretamente com pior qualidade de vida $^{(20)}$. No presente estudo, as implicações da qualidade de vida são visualizadas pela alta frequência de pacientes em fase terminal, alta presença de complicações e comorbidades associadas, baixa TFG e maior frequência de pacientes com excesso de peso. Em partes, isso pode ser justificado porque pacientes com renda limitada e baixa escolaridade possui inadequado nível de letramento funcional em saúde, resultando em dificuldades ao processar as informações em saúde recebidas, transformá-las em conhecimento e assim, aplicá-las no autogerenciamento da doença ${ }^{(21)}$.

Os dados sobre incidência e prevalência da doença renal crônica não dialítica (DRC-ND) ainda são escassos, entretanto, já estão bem documentados na literatura esses dados sobre pacientes em terapia renal substitutiva (TRS). Quando analisados os dados sobre o censo de 2012 dos pacientes em TRS mostrou que a doença de base para o diagnóstico da DRC foi em $34 \%$ HAS e $29 \% \mathrm{DM}^{(22)}$. Nossos resultados demonstram tendência semelhante, sugerindo que a nefroesclerose hipertensiva seguida da nefropatia diabética foram as causas principais para a DRC nos pacientes deste estudo.

$\mathrm{O}$ número pequeno de pacientes incluídos em nosso estudo e a alta prevalência em fase terminal da doença pode ser explicado em parte porque há baixa efetividade no rastreamento da DRC na atenção primária a saúde, onde com frequência a DRC é subdiagnosticada devido à desatualização ou falta de informação com relação à DRC dos profissionais atuantes na atenção básica ${ }^{(23)}$.

Alguns parâmetros importantes avaliados em nosso estudo foram urina espumosa e trato gastrointestinal irregular, a expressão mútua desses sinais e sintomas se traduzem na síndrome urêmica, que provoca importantes consequências deletérias no estado nutricional do paciente, e mais 
de $1 / 3$ dos nossos pacientes apresentaram uremia.

A obesidade recentemente foi associada como fator independente para desenvolver DRC, isso porque provocam alterações hemodinâmicas, estruturais, histológicas, assim como, desordens metabólicas e bioquímicas que cursam com a agressão renal $^{(5)}$, além disso, sabe-se que o tecido adiposo é metabolicamente ativo, produzindo adipocinas que por mecanismos diversos influenciam nas respostas de diferentes tecidos e órgãos. Sugere-se que os mecanismos pelos quais a obesidade incremente a incidência e progressão da DRC seja pela resistência à insulina, inflamação, estresse oxidativo, hiperlipidemia e disfunção endotelial $^{(24)}$.

Em nosso estudo, a avaliação do estado nutricional segundo o IMC revelou alta prevalência de pacientes com excesso de peso, contudo, um fator limitante para essa avaliação foi a presença de edema em alguns pacientes. Entretanto, a alta prevalência de obesidade foi confirmada pelas avaliações realizadas através $\mathrm{CC} \quad \mathrm{e}$ $\% \mathrm{GC}$.

Como citado anteriormente, a avaliação da obesidade utilizando apenas o IMC possui limitações, pois esse índice não é capaz de fornecer informações relacionadas à distribuição e quantidade de gordura corporal, então adicional a esse índice, nesse estudo para avaliação da obesidade, foi utilizado a $\mathrm{CC}$ e $\% \mathrm{GC}$, pois avaliam as limitações existentes no IMC.

A avaliação da obesidade central foi realizada pela $\mathrm{CC}$ e mostrou alta prevalência. A obesidade central tem efeitos deletérios em cascata sobre os rins, ela provoca aumento da pressão abdominal, gerando maior compressão renal e como consequência maior pressão intrarrenal, acelerando assim a progressão da DRC. A obesidade central no paciente com DRC também tem associação com inflamação, resistência à insulina, dislipidemia, estresse oxidativo, eventos cardiovasculares e mortalidade. Adicional a esses efeitos deletérios citados, a obesidade central confere risco adicional aos pacientes com DRC para desfechos cardiovasculares ${ }^{(5,25)}$.

$\mathrm{O}$ excesso de tecido adiposo em nossa amostra foi confirmado também pela \%GC alta entre nossos pacientes. $\mathrm{Na}$ obesidade estão presentes níveis altos de leptina e baixos de adiponectina, essas duas adipocinas convergem em uma ação que piora o quadro dos pacientes com DRC, elas deixam o sistema nervoso simpático renal (SNSR) superativado, e este 
sinaliza para que ocorra maior reabsorção tubular de sódio, prejudicando assim a natriurese. Essa reabsorção de sódio promove maior retenção hídrica e assim aumento dos níveis pressóricos ${ }^{(25)}$.

Não foi possível realizar análises para determinar se há associação entre os estágios de DRC e as variáveis antropométricas, pois nossa amostra foi pequena para realização desta análise. Contudo, quando comparadas às médias dos grupos com adequado e alto $\% \mathrm{GC}$ e as variáveis antropométricas (IMC, CB, $\% \mathrm{CB}$ ), creatinina e $\mathrm{TFG}$ foram observadas diferenças estatisticamente significativas $(\mathrm{p}<0,05)$, onde o grupo com \%GC alto obteve médias maiores do que aqueles no grupo com adequado $\%$ GC, com descrito na tabela 4 .

Os mecanismos da obesidade que provocam maior reabsorção de sódio induz a um aumento do fluxo plasmático promovendo expansão, tendo como consequência o aumento do fluxo sanguíneo renal, o aumento da TFG e na fração filtrada ${ }^{(25)}$. Inicialmente é perceptível essa hiperfiltração glomerular nos obesos, onde os pacientes com maior \%GC obtiveram TFG maiores que aqueles com adequada $\% \mathrm{GC}$, como observamos na tabela 4. Contudo, essa hiperfiltração em longo prazo provoca lesões nos glomérulos, tendo como consequência final a glomerulosclerose global, que dará início junto com outros mecanismos a um declínio progressivo da TFG e ao desencadeando da DRC.

Nosso estudo obteve como fator limitante a amostra reduzida, o que inviabilizou a realização de testes que demonstrassem associações entre os estágios da DRC e variáveis antropométricas. Por isso recomenda-se que novos estudos com amostras mais robusta venham a ser realizados para estabelecer possíveis associações.

\section{Conclusão}

A pesquisa permitiu conhecer o perfil antropométrico dos portadores de DRC em tratamento conservador em nosso ambulatório, onde a obesidade foi mais prevalente. Os achados reforçam a necessidade da implementação de intervenções mais eficazes para o controle das comorbidades associadas à DRC e, principalmente o cuidado para manutenção de um peso adequado, evitando complicações no tratamento devido à obesidade.

Outro fator relevante é que a avaliação do estado nutricional deve ser realizada não somente por apenas um método, mas por um conjunto de medidas, índices e adequações, para que 
toda a dimensão do pacientes seja compreendida e para que a intervenção individualizada possa ser realizada.

\section{Referências}

1. World Health Organization. Global status report on noncommunicable diseases 2014. Geneva; 2014: 302.

2. World Health Organization. Noncommunicable Diseases Progress Monitor 2015. Geneve; 2015: 236.

3. Jha $\mathrm{V}$, Garcia-Garcia $\mathrm{G}$, Iseki $\mathrm{K}$, Li Z, Naicker S, Plattner B, et al. Chronic kidney disease: global dimension and perspectives. Lancet; 2013;382;(9888): 260-72.

4. Ministério da Saúde (BR). Vigitel Brasil 2016: Vigilância de Fatores de Risco e Proteção para Doenças Crônicas por Inquérito Telefônico: Estimativas sobre frequência $\mathrm{e}$ distribuição sociodemográfica de fatores de risco e proteção para doenças crônicas nas capitais dos 26 estados b. Brasília: Ministério da Saúde; 2017.

5. Kopple JD, Feroze U. The Effect of Obesity on Chronic Kidney Disease. J. Nutrição [periódico na internet]. 2011 [citado 2018 mar. 17]; 21(1): 66-1. Disponível em: https://www.ncbi.nlm .nih.gov/pubmed/21195923.

6. Cipullo JP, Martin JFV, Ciorlia LA de S, Godoy MRP de, Cação JC, Loureiro AAC, et al. Prevalência e fatores de risco para hipertensão em uma população urbana brasileira. Cardiologia (São Paulo) [periódico na internet]. 2010 [citado 2017 ago. 19]; 94(4):519-26. Disponível em: http://www.scielo.br/pdf/abc/v94n4/ao p00810.pdf.
7. Agrawal V, Giri C, Solomon RJ. The Effects of Glucose-Lowering Therapies on Diabetic Kidney Disease. Diabetes [periódico na internet]. 2015 [citado 2018 mai. 1]; 11(3): 191-00. Disponível em: https://www.ncbi.nlm. nih.gov/pubmed/25824237.

8. Guyatt GH, Oxman AD, Kunz R, Al. E. KDIGO 2017 Clinical Practice Guideline Update for the Diagnosis, Evaluation, Prevention, and Treatment of Chronic Kidney Disease-Mineral and Bone Disorder (CKD-MBD). Kidney Int Suppl. 2017;7(1):1-59.

9. Anderson CAM, Nguyen HA, Rifkin DE. Nutrition Interventions in Chronic Kidney Disease. Medicine [periódico na internet]. 2016 [citado 2017 set. 22]; 100(6): 1265-83. Disponível em: https://www.ncbi.nlm. nih.gov/pubmed/27745594.

10. Ferreira MG, Sichieri R. Epidemiologia nutricional. In: Gilberto K, Rosely S, Denise Petrucci G, Organizadores. Antropometria como método de avaliação do estado de nutrição e saúde do adulto. Rio de Janeiro: FIOCRUZ/Atheneu; 2007; 5: 93-04. http://books.scielo.org/id/rrw5 w/08.

11. Barros MBDA, Francisco PMSB, Zanchetta LM, César CLG. Tendências das desigualdades sociais e demográficas na prevalência de doenças crônicas no Brasil, PNAD: 2003- 2008. Saúde Coletiva (Rio de Janeiro) [periódico na internet]. 2011 [citado 2017 nov. 3]; 16(9):3755-68. Disponível em: http://www.scielo.br/ scielo.php?script $=$ sci_arttext\&pid $=\mathrm{S} 14$ 13-81232011001000012. 
12. Chumlea WC, Roche AF, Steinbaugh ML. Estimating Stature from Knee Height for Persons 60 to 90 Years of Age. Geriatria [periódico na internet]. 1985 [citado 2017 dez. 11]; 33(2):116-20. Disponível em: https://www.ncbi.nlm.nih.gov/pubmed/ 3968366.

13. World Health Organization. Physical status: the use and interpretation of anthropometry. Report of a WHO Expert Committee. WHO Tech repost Ser. 1995;1-452.

14. Padovani CS da S. Avaliação do perfil epidemiológico e das dificuldades encontradas pelos pacientes para $\mathrm{o}$ atendimento de primeira consulta no ambulatório de triagem da nefrologia da UNIFESP. J Nefrologia (São Paulo) [periódico na internet]. 2012 [citado 2018 jan. 25]; 34(4):317-22. Disponível em: https://www.ncbi.nlm.nih.gov/pubmed/ 3968366.

15. Cabral PC, Diniz ADS, Arruda IKG De. Avaliação nutricional de pacientes em hemodiálise. Nutrição (Campinas) [periódico na internet]. 2005 [citado 2017 ago. 22]; 18(1):290. Disponível em: http://www.scielo .br/pdf/rn/v18n1/23505.pdf.

16. Brandão AP, Brandão, Andréa Araujo Nogueira, Armando da Rocha Suplicy H, Guimarães JI, Oliveira JEP de. I Diretriz Brasileira de Diagnóstico e Tratamento da Síndrome Metabólica. Cardiologia (Rio de Janeiro). [periódico na internet]. 2005 [citado 2017 set. 30]; 84(1):1-28. Disponível em: http://publicacoes.cardiol.br/conse nso/2005/sindromemetabolica.pdf.
17. Deurenberg $\mathrm{P}$, Weststrate JA, Seidell JC. Body mass index as a measure of body fatness: age- and sexspecific prediction formulas. Nutrição. [periódico na internet]. 1991 [citado 2018 fev. 12]; 65(2):105. Disponível em: https://www.ncbi.nlm.nih.gov/pub $\mathrm{med} / 2043597$.

18. Leal VDO, Leite Júnior M, Mafra D. Acidose metabólica na doença renal crônica: abordagem nutricional. Nutrição (Campinas). [periódico na internet]. 2008 [citado 2017 jul. 20]; 21(1):93-03. Disponível em: http://www.scielo.br/scielo.php?script $=$ sci_arttext\&pid $=\mathrm{S} 1415-5273200800$ 0100010 .

19. Sociedade Brasileira de Nefrologia. Diretrizes Clínicas Para o Cuidado ao Paciente com Doença Renal Crônica - DRC no Sistema Único de Saúde. Assoc Médica Bras. 2014;37.

20. Porter A, Fischer MJ, Brooks D, Bruce M, Charleston J, Cleveland WH, et al. Quality of life and psychosocial factors in African Americans with hypertensive chronic kidney disease. Transl Res. 2012;159(1):4-11.

21. Moraes KL, Brasil VV, Oliveira GF de, Cordeiro JABL, Silva AMTC, Boaventura RP, et al. Letramento funcional em saúde e conhecimento de doentes renais em tratamento prédialítico. Enfermagem (Brasília). [periódico na internet]. 2017 [citado 2018 fev. 1]; 70(1):155-62. Disponível em: http://www.scielo.br/pdf/reben/v 70n1/0034-7167-reben-70-01-0155. pdf. 
22. Sesso RC, Lopes AA, Thomé FS, Lugon JR, Watanabe Y, Santos DR dos. Relatório do Censo Brasileiro de Diálise Crônica 2012. J Bras Nefrol. 2014;36(1):48-53.

23. Paula EA de, Costa MB, Colugnati FAB, Bastos RMR, Vanelli CP, Leite CCA, et al. Potencialidades da atenção primária à saúde no cuidado à doença renal crônica. Rev Lat Am Enfermagem. 2016;24(e2801):1-9.

24. Ting SMS, Nair H, Ching I, Taheri S, Dasgupta I. Overweight, Obesity and Chronic Kidney Disease. Nephron Clin Pract. 2009;112(3):c121-7.

25. D'Agati VD, Chagnac A, de Vries APJ, Levi M, Porrini E, HermanEdelstein M, et al. Obesity-related glomerulopathy: clinical and pathologic characteristics and pathogenesis. Nat Rev Nephrol. 2016;12(8):453-71. 